\title{
Perspectiva dos Discentes de Medicina de uma Universidade Pública sobre Saúde e Qualidade de Vida
}

\author{
Viewpoint of Medical Students from a Public \\ University on Health and Quality of Life
}

\author{
Angela Maria Moreira Canuto Mendonça ${ }^{I \oplus}$ \\ Thaís Ferreira Gêda \\ Julia Espíndola Guimarães ${ }^{I}$ \\ Caroline Oliveira Mendes ${ }^{I}$ \\ Tharnier Barbosa Franco Manna ${ }^{I}$ \\ Eduardo Maffra Monteiro
}

\section{PALAVRAS-CHAVE}

- Estudantes de Medicina.

- Qualidade de Vida.

- Sinais e Sintomas Digestórios.

- Condições Patológicas, Sinais e Sintomas.

\section{RESUMO}

Introdução: Desde o preparo para o ingresso até a conclusão do curso de Medicina, há uma cobrança além do conteúdo; exige-se preparo emocional. Diante de um curso com carga horária integral, que demanda dedicação intensa para atingir boa bagagem teórico-prática e contemplar as exigências curriculares, e, muitas vezes, da distância da família, tem-se a percepção diminuída da qualidade de vida entre esse grupo. Essa adaptação a mudanças fisiológicas e emocionais é capaz de interferir no processo de adoecimento desse grupo, refletindo na atuação profissional e na comunidade em que atuará. Objetivo: Conhecer hábitos de vida e o processo de adoecimento dos acadêmicos de Medicina da Universidade Federal de Alagoas (UFAL) ao longo do curso, além de buscar entender a possível relação entre esses fatores e a alta incidência de queixas gástricas nesse grupo. Metodologia: Estudo descritivo e transversal cuja amostra é composta de estudantes de Medicina do 1o ao 8o período da UFAL, separados por gênero e ciclo do curso. Utilizou-se o método de Bardin para análise qualitativa do conteúdo gerado na discussão dos grupos. Resultados: Por meio da análise de Bardin, chegou-se à categoria final qualidade de vida, em todos os grupos, feminino e masculino, tanto do ciclo básico quanto do ciclo clínico. Essa categoria advém das categoriais intermediárias: acessibilidade à alimentação saudável, hábitos de vida e sintomas gerais. A carga horária exorbitante foi a principal queixa dos grupos. Chamaram atenção a frequência das queixas gastrointestinais e a distância do núcleo familiar. Destacaram-se as peculiaridades de cada grupo nas categoriais iniciais, a competitividade entre as alunas do $5^{0}$ ao $8^{\underline{a}}$ período do sexo feminino e a ingestão de bebida alcoólica entre alunos do sexo masculino do $5^{0}$ e o $8^{\circ}$ período. Discussão: A diminuição da qualidade de vida é resultado da matriz curricular, com mais de oito mil horas. $O$ reflexo dessa carga horária exorbitante é o estresse, que serve de gatilho para qualidade de sono ruim e carência de tempo para dormir, hábitos alimentares precários, ingestão de bebida alcoólica, nervosismo, ansiedade, competividade, sintomas como cefaleia, dores nas costas e sintomas e patologias digestivas. Com o sistema de seleção unificado, a distância do núcleo familiar transformou o perfil e as necessidades desse grupo, refletindo nas condições financeiras e responsabilidades adicionais que precisam assumir. Considerações finais: Os resultados encontrados nesta pesquisa sobre os principais fatores que levam à diminuição da qualidade de vida e à repercussão desse processo no adoecimento tanto de estudantes do sexo masculino quanto do feminino nos diferentes períodos do curso de Medicina coincidem com os da literatura nacional e internacional. Há uma tendência mundial que aposta no autocuidado do currículo médico, a fim de formar profissionais mais capacitados. Em vista disso, este artigo pode despertar as instituições universitárias sobre a necessidade de intervir no curso de Medicina. 


\section{KEY-WORDS}

- Medical Students.

- Health-Related Quality of Life.

- Digestive System Disease.

- Symptoms and General Pathology.

\begin{abstract}
Introduction: From premed to graduating from medical school, more than just the academic content is required from students, and emotional preparation is necessary. Faced with a full-time course that demands intense dedication to achieve good theoretical-practical background and contemplate the curricular requirements and, being often far away from one's family, there is a diminished perception of quality of life in this group. This adaptation to physiological and emotional changes is capable of interfering with the disease process in this group of individuals, affecting their professional performance and the community where they will work at. Objective: To know the life habits and disease processes of medical students of the Federal University of Alagoas throughout the medical course. In addition to seeking to understand the possible association between these factors and the high incidence of gastric complaints in this group. Methodology: This is a descriptive and crosssectional study, comprising medical students from the $1^{\text {st }}$ to $8^{\text {th }}$ periods of the Federal University of Alagoas, separated by gender and course cycle. The Bardin method was used for the qualitative analysis of the content generated during the discussions with the groups. Results: Through Bardin analysis, the final category of quality of life was reached in the female and male groups, both in the basic cycle and in the clinical cycle. This category derives from intermediate categories: access to a healthy diet, life habits and overall symptoms. An excessive workload was the groups' main complaint. The frequency of gastrointestinal complaints and those related to being away from the family is noteworthy. It is worth noting the peculiarities of each group in the initial categories, the competitiveness among female students from the $5^{\text {th }}$ to the $8^{\text {th }}$ periods and alcohol consumption among male students in the $5^{\text {th }}$ and $8^{\text {th }}$ periods. Discussion: The decrease in the quality of life is a result of the curricular matrix with more than 8,000 hours. The consequences of this excessive workload is the stress that works as a trigger for poor sleep quality and lack of time to sleep; unhealthy eating habits; alcohol consumption; nervousness, anxiety, competitiveness; symptoms such as headaches, back pain and digestive symptoms and pathologies. With the Unified Selection System, being away from the family nucleus has transformed the profile and needs of this group, reflecting the financial conditions and additional responsibilities they need to assume. Final Considerations: The results of this research about the main factors that lead to a decrease in the quality of life and the consequences of this process for the health of both male and female students during the different periods of medical school are in agreement with the national and international literature. There is a worldwide tendency that contemplates including self-care in the medical school curriculum, aiming to train more qualified professionals; hence, this article can make the university institutions aware of the need to intervene in the medical course.
\end{abstract}

\section{INTRODUÇÃO}

O curso de Medicina, no Brasil, desperta muito interesse entre os candidatos a um curso superior. Por isso, a profissão médica já se inicia com uma seleção muito concorrida e que exige preparo intelectual e emocional, a fim de lidar com expectativas individuais e coletivas. As pressões da família, a necessidade de autoafirmação e as exigências sociais levam o indivíduo, muito jovem, a se privar de diversos interesses com o propósito de conseguir o objetivo: ingressar na graduação do curso de Medicina. No entanto, conquistado esse objetivo, não cessam as dificuldades enfrentadas pelos estudantes ${ }^{1}$.

Durante o curso, a percepção de qualidade de vida pode diminuir para corresponder a uma formação que exige horário integral e boa bagagem teórico-prática. O estudante, logo nos primeiros meses do curso, depara com uma série de situações desafiadoras e estressantes: a carga horária em tempo integral, o confronto diário com experiências de doença e morte e, muitas vezes, a distância da família. Diante de tantos desafios, o 
acadêmico de Medicina poderá ser dominado pelo cansaço, pela tristeza e pela insegurança e até mesmo por patologias psicossomáticas, o que parece ter como consequência mudanças fisiológicas e emocionais. Todas essas transformações exigem capacidade de adaptação a um novo estilo de vida, a fim de evitar o processo de adoecimento ${ }^{1}$. As experiências vivenciadas pelo estudante de Medicina refletem-se no profissional que ele se tornará, o que afeta não apenas a sua formação, mas também a comunidade na qual ele atuará ${ }^{2,3}$.

Esta pesquisa, qualitativa e baseada em grupos focais, teve como intuito conhecer esses hábitos de vida e o processo de adoecimento - como se transformaram e/ou ocorreu, respectivamente - ao longo do curso. Assim, tais resultados podem despertar as instituições universitárias a respeito da necessidade de intervir no curso de Medicina.

\section{METODOLOGIA}

Este estudo teve desenho descritivo e transversal com avaliação qualitativa e utilizou grupos focais. A amostragem foi randomizada por conveniência com base nos indivíduos do $1^{\underline{o}}$ ao $8^{\underline{o}}$ período do curso de Medicina da Universidade Federal de Alagoas (UFAL) que atenderam ao critério de inclusão aceitação voluntária. Foram excluídos aqueles que não aceitaram participar da pesquisa ou desistiram de fazer parte dela durante o período da realização dos grupos focais.

A análise do conteúdo dos grupos focais é identificada como uma das mais adequadas para o acesso a relatos e reflexões dos estudantes sobre a sua alimentação, queixas gástricas e hábitos de vida. Representa uma fonte que intensifica o acesso às informações acerca de um fenômeno, seja pela possibilidade de gerar novas concepções, seja pela análise e problematização de uma ideia em profundidade. Além disso, esse método permite maior interação entre os participantes ${ }^{4}$.

A discussão foi guiada por perguntas semiestruturadas dirigidas pelo mediador, a fim de sensibilizar os participantes para operar na transformação da realidade de modo crítico e criativo, ao permitir a análise objetiva e subjetiva e também a livre expressão e participação ${ }^{5}$. O roteiro foi composto de oito perguntas, que norteiam a discussão:

1. Como conciliar os horários no curso de Medicina?

2. Como é a alimentação de vocês no momento?

3. Há relação entre estresse e curso de Medicina?

4. Qual é a relação entre o estilo de vida, a alimentação e o curso de Medicina?

5. Há alguma relação entre problemas físicos/psíquicos e o curso de Medicina?

6. O curso de Medicina permite que vocês tenham espaços de lazer?
7. Vocês têm sintomas gástricos ou outros quaisquer relacionados ao curso de Medicina?

8. Vocês utilizam medicamentos após o início do curso de Medicina?

As reuniões entre os grupos ocorreram na Faculdade de Medicina (Famed) em encontros semanais com duração de aproximadamente 40 a 60 minutos. Os pesquisadores foram divididos nas funções de mediador, que coordenava a participação dos integrantes; observador, responsável por fazer anotações pertinentes sobre expressão corporal e emoções; e um terceiro, com a incumbência de gravar a discussão posteriormente transcrita para análise de dados.

De acordo com a literatura, a quantidade mínima de participantes por grupo focal é de 8-12 indivíduos. Os grupos desta pesquisa foram constituídos de uma média de nove pessoas - foram quatro grupos e 34 participantes no total. Estes foram divididos por gênero, com o objetivo de analisar se existe interferência do sexo, e ainda separados por ciclo em que se encontram - ciclo básico, do $1^{\circ}$ ao $4^{\circ}$ período; e ciclo clínico, do $5^{\mathrm{o}}$ ao $8^{\mathrm{o}}$.

O método de análise de conteúdo escolhido foi o de Bardin, pelas etapas organizadas em:

- pré-análise;

- exploração material;

- tratamento dos resultados, inferência e interpretação.

A fase de pré-análise compreende a leitura do material transcrito, a fim de definir o corpus de análise, formular hipóteses e objetivos e escolher os indicadores. A seleção dos dados é feita com base na exaustividade, representatividade, homogeneidade e pertinência. Durante a fase de exploração do material, fazem-se a eleição das unidades de registro e a formação das categorias simbólicas e temáticas que serão apresentadas na discussão. Por fim, tem-se a interpretação, fase em que são apontadas as inferências com base nas categorias criadas ${ }^{6,7}$.

De acordo com a Resolução no 466/2012 do Conselho Nacional de Saúde, foi aplicado o Termo de Consentimento Livre e Esclarecido a todos os participantes. Os dados coletados serão mantidos sob sigilo com os pesquisadores, excetuando-se as informações que puderem servir no processo de intervenção do curso, mas mantendo-se o anonimato dos pesquisados. A pesquisa foi submetida à Plataforma Brasil com Certificado de apresentação para Apreciação Ética (CAAE) no 71391417.0.0000.5013. 


\section{RESULTADOS}

Por meio da análise de Bardin dos resultados encontrados nos grupos focais, foram identificadas categorias iniciais, intermediárias e final.

Nos grupos masculinos, chegaram-se às categorias iniciais tempo e morar sozinho no ciclo básico e no clínico. Já excesso de atividades foi vista apenas no ciclo básico; e fila no restaurante universitário (RU), somente no ciclo clínico. Essas categorias foram agrupadas na categoria intermediária acessibilidade à alimentação saudável: "Então eu tô comendo todo dia no mesmo horário e estou comendo bem, sim, apesar de que o problema lá da fila é absurdo" (homem, ciclo clínico).

Ainda no grupo masculino do ciclo básico, constataram-se as categorias iniciais pouco lazer, sono e alimentação irregular. No ciclo clínico, alimentação irregular, exercício físico, sono e etilismo foram agrupadas na categoria intermediária hábitos de vida: "Meu estilo de vida mudou bastante, porque eu nunca consigo dormir as sete horas por dia que eu preciso dormir, e isso impactou na qualidade de vida dos estudos e do lazer e também na alimentação" (homem, ciclo clínico)

Se a cerveja está muito barata no mercado, aí você pensa eu vou comprar. [...] Aí eu penso que estou estressado..., cansado dessa rotina, pensando em sair um pouco. Aí vai curtir, vai beber e tal, bebe no domingo, bebe na sexta. Isso atrapalha. Final de semana você deveria estar estudando alguma coisa e aí não está e atrapalha (homem, ciclo clínico).

Já as categorias iniciais refluxo, dermatite, enxaqueca, arritmia cardíaca, dor abdominal, estresse, epigastralgia, ansiedade e insônia foram encontradas no ciclo básico e no ciclo clínico. Epigastralgia, diarreia, dificuldade de concentração, dor nas costas, insônia, nervosismo e ansiedade foram englobadas na categoria intermediária sintomas gerais: "Mas eu comecei a sentir esse refluxo agora, na entrada no curso de Medicina. [...] Muitas já me peguei assim taquicárdico em questão de ansiedade, de ficar hiperventilando" (homem, ciclo básico).

Como categoria final comum a todos os grupos, observou-se qualidade de vida.

Nos grupos femininos, as categorias iniciais tempo e morar sozinho foram relatadas no ciclo clínico, enquanto no ciclo básico foram as mesmas, tempo e o fato de morar sozinho, acrescidas do excesso de atividades. Elas foram reunidas na categoria intermediária acessibilidade à alimentação saudável: "Se eu gasto tempo comendo, considero tempo perdido. Deveria estar estudando" (mulher, ciclo clínico).

Como categoria inicial, o ciclo básico referiu-se à alimentação irregular e a exercício físico, e o ciclo clínico, a exercício físico e sono, as quais foram agrupadas na categoria intermediária hábitos de vida. Na categoria intermediária sintomas gerais, o ciclo básico abordou como categorias iniciais náusea, dermatite, sonolência, estresse, epigastralgia, refluxo, gastrite, ansiedade, pirose, cefaleia e insônia. Por outro lado, o ciclo clínico englobou diarreia, preocupação com notas, frustração, estresse, culpa e ansiedade: “Eu tenho uns quadros de azia. [...] De manhã eu tenho bastante dor na barriga. É na barriga inteira. [...] Foi depois do curso. Acho que a primeira vez eu estava no segundo período e já aconteceu várias vezes" (mulher, ciclo básico); "Você não suporta tirar uma nota baixa. Eu digo isso por mim, eu odeio tirar nota baixa. Agora, tipo, eu sei que eu vou ficar triste. Então para eu não ficar triste depois, eu fico triste agora e estudo" (mulher, ciclo clínico).

Como categoria final comum a todos os grupos, obteve-se qualidade de vida.

Não houve disparidade entre as categorias intermediárias dos grupos feminino e masculino, sendo as três principais: acessibilidade à alimentação saudável, hábitos de vida e sintomas gerais (Quadros 1 e 2). Como categoria final, apresentaram-se os aspectos da vida do estudante de Medicina, os quais interferem na qualidade de vida (Quadros 1 e 2).

\section{DISCUSSÃO}

Neste estudo, pôde-se observar um relato de percepção diminuída de qualidade de vida, o que se deve, entre muitos aspectos, à carga horária elevada do curso em questão. O projeto pedagógico do curso (PPC) de Medicina da UFAL divide-se em ciclo básico, ciclo clínico e internato. A matriz curricular é modular e constituída de aulas teóricas e práticas distribuídas pela manhã, tarde e eventualmente noite, de segunda a sábado. Além disso, tem-se as metodologias ativas, como a tutoria do $1^{\circ}$ ao $4^{\circ}$ período, uma competência acadêmica capaz de atender às demandas atuais e às diretrizes curriculares nacionais com domínio dos fundamentos que sustentam as bases do conhecimento ${ }^{8}$. Há cerca de duas tardes por semana da organização curricular com tempo livre. Estas, segundo o PPC, destinam-se à incorporação de outras formas de aprendizagem em atividades complementares e disciplinas eletivas, compondo a parte flexível do curso, que deverá atingir até 5\% da carga horária obrigatória. Esses tempos livres, no entanto, nem sempre são respeitados pelos docentes.

No que se refere a todos os períodos do $1^{\circ}$ ao $4^{\circ}$, verifica-se que os alunos têm reclamações semelhantes, independentemente de gênero. $\mathrm{O}$ excesso de atividades curriculares reflete no gerenciamento do tempo, sobretudo para lazer, exercícios físicos, alimentação e sono. A esse respeito, estudos afirmam que o excesso de atividades na universidade pode influenciar 


\begin{tabular}{|c|c|c|c|}
\hline \multirow{3}{*}{ CATEGORIA FINAL } & \multicolumn{3}{|c|}{$\begin{array}{l}\text { QUADRO } 1 \\
\text { Distribuição das categorias dos grupos focais masculinos }\end{array}$} \\
\hline & \multirow{2}{*}{$\begin{array}{l}\text { CATEGORIAS } \\
\text { INTERMEDIÁRIAS }\end{array}$} & \multicolumn{2}{|c|}{ CATEGORIAS INICIAIS } \\
\hline & & CICLO BÁSICO & CICLO CLÍNICO \\
\hline \multirow{3}{*}{ Qualidade de vida } & $\begin{array}{c}\text { ACESSIBILIDADE A UMA } \\
\text { ALIMENTAÇÃO SAUDÁVEL }\end{array}$ & $\begin{array}{l}\text { Tempo, Morar sozinho,Excesso de } \\
\text { atividades }\end{array}$ & $\begin{array}{c}\text { Tempo, Morar sozinho Fila No } \\
\text { Ru, }\end{array}$ \\
\hline & HÁBITOS DE VIDA & $\begin{array}{l}\text { Pouco Lazer, Sono, Alimentação } \\
\text { Irregular }\end{array}$ & $\begin{array}{l}\text { Alimentação Irregular, Exercício } \\
\text { Físico, Sono, Etilismo }\end{array}$ \\
\hline & SINTOMAS GERAIS & $\begin{array}{l}\text { Refluxo, Dermatite, Enxaqueca, } \\
\text { Arritmia Cardíaca, Dor } \\
\text { Abdominal, Estresse, } \\
\text { Epigastralgia, Ansiedade, Insônia }\end{array}$ & $\begin{array}{l}\text { Epigastralgia, Diarreia, } \\
\text { Dificuldade de Concentração, Dor } \\
\text { nas Costas, Insônia, Nervosismo, } \\
\text { Ansiedade }\end{array}$ \\
\hline
\end{tabular}

\begin{tabular}{|c|c|c|c|}
\hline \multicolumn{4}{|c|}{$\begin{array}{c}\text { QUADRO } 2 \\
\text { Distribuição das categorias dos grupos focais femininos }\end{array}$} \\
\hline \multirow{2}{*}{ CATEGORIA FINAL } & \multirow{2}{*}{$\begin{array}{l}\text { CATEGORIAS } \\
\text { INTERMEDIÁRIAS }\end{array}$} & \multicolumn{2}{|c|}{ CATEGORIAS INICIAIS } \\
\hline & & CICLO BÁSICO & CICLO CLÍNICO \\
\hline \multirow{3}{*}{ Qualidade de vida } & $\begin{array}{c}\text { ACESSIBILIDADE A UMA } \\
\text { ALIMENTAÇÃO SAUDÁVEL }\end{array}$ & $\begin{array}{c}\text { Tempo, Morar Sozinho, Excesso } \\
\text { de Atividades, Custos }\end{array}$ & Tempo, Morar Sozinho, Custos \\
\hline & HÁBITOS DE VIDA & $\begin{array}{c}\text { Exercicio Físico, Alimentação } \\
\text { Irregular }\end{array}$ & Exercício Físico, Sono \\
\hline & SINTOMAS GERAIS & $\begin{array}{c}\text { Nausea, Dermatite, Sonolência, } \\
\text { Estresse, Epigastralgia, Refluxo, } \\
\text { Gastrite, Ansiedade, Pirose, } \\
\text { Cefaleia, Insônia }\end{array}$ & $\begin{array}{l}\text { Diarreia, Preocupação com Notas, } \\
\text { Frustração, Estresse, Culpa, } \\
\text { Ansiedade }\end{array}$ \\
\hline
\end{tabular}

de maneira negativa a vida de estudantes de Medicina e destacam prejuízos nessa rotina ${ }^{9,10}$. Assim, os dados apresentados sobre as mais de oito mil horas da matriz curricular constituem a principal queixa dos alunos: a carga horária exorbitante.

Desde 2011, a UFAL aderiu ao Sistema de Seleção Unificado (SiSU), como sugerido pelo Ministério da Educação. Com isso, houve uma mudança no perfil dos alunos que compõem o quadro discente da instituição. Dessa forma, as necessidades pessoais desse grupo relacionam-se também com a distância do núcleo familiar, como identificado nas categorias iniciais dos grupos. Isso reflete nas condições financeiras, visto que, grande parte dos alunos da FAMED não têm renda e os gastos são financiados pela família ou por outras pessoas. Comparando-se os gêneros, não houve diferença, com exceção da maior preocupação com as questões financeiras por parte do sexo feminino. A condição psicossocial também é importante, já que os alunos não dispõem de suporte às responsabilidades nas diversas atividades domésticas.

No tocante à alimentação, perceberam-se hábitos alimentares precários citados em todo o universo pesquisado, desde a dificuldade em enfrentar filas no RU até os altos preços cobrados pelo restaurante da própria Famed, incompatíveis com a situação econômica da maioria dos estudantes. A respeito da ingesta nutricional, evidencia-se que os jovens, incluindo universitários da área da saúde, apresentam características pouco saudáveis. Entre elas, vê-se o excesso de consumo de sal, de gorduras saturadas e de açúcares procedentes especialmente de alimentos processados e bebidas adocicadas ${ }^{11-13}$. A maioria desses estudantes informou trocar o almoço ou jantar por lanches rápidos.

De acordo com pesquisa que relacionou câncer e doenças cardiovasculares com a dieta, a contribuição para a compreensão da importância da educação dos estudantes de Medicina na área da nutrição é fundamental ${ }^{14}$, já que é peça-chave na promoção e prevenção de saúde. Os resultados confirmam que conhecimento pode ser transformado em alimentação saudável e ser utilizado para que os estudantes entendam a sua própria dieta e, assim, a aprimorem, contribuindo com a oportunidade de agir como modelo no aconselhamento dos pacientes ${ }^{14,15}$.

Entre o $5^{\circ}$ e o $8^{\circ}$ período, aparece no discurso dos grupos masculinos estudados a ingestão de bebida alcoólica como maneira de relaxar após as atividades acadêmicas. Chama a atenção o fato de alguns estudantes afirmarem que começaram a ingerir bebidas alcoólicas, em geral cerveja, dependendo da acessibilidade e do preço, durante a semana, e não apenas 
nos fins de semana. Estudos realizados pelas Universidade de São Paulo, Marília e do Maranhão confirmam o alto índice de consumo de álcool entre os estudantes, podendo chegar a 64,2\% no Maranhão, 80,1\% em Marília e até 89,3\% em São Paulo ${ }^{16-18}$. Esse consumo excessivo está relacionado à queda no desempenho acadêmico, ao prejuízo no desenvolvimento e na estruturação de habilidades cognitivo-comportamentais e emocionais, a danos ao patrimônio público e à violência ${ }^{19}$.

Estudos prospectivos atestam a piora da qualidade do sono de acordo com a progressão do curso, entre o $4^{\circ}$ e o $7^{\circ}$ semestre $^{20-22}$. Outros demonstram que em diferentes universidades americanas a média de horas de sono diminui de forma significante do ingresso da faculdade até as práticas/os plantões e o último ano da graduação ${ }^{23}$. Os resultados desta pesquisa, em relação à qualidade do sono e à carência de tempo para dormir, condizem com a literatura mundial, contudo não se verificaram variações significativas entre os períodos estudados. Isso pode ser explicado talvez pelo fato de esta pesquisa não ter contemplado alunos do internato ( $9^{\circ}$ ao $12^{\circ}$ semestres), em que habitualmente as práticas de plantão são mais frequentes.

Em todos os grupos focais desta investigação, independentemente do período e do gênero, relatos de nervosismo, ansiedade, estresse e distúrbios do sono foram comuns. Chamaram a atenção entre as alunas do $5^{\circ}$ ao $8^{\circ}$ período do sexo feminino a competitividade e o sentimento de frustração e de culpa, caso estejam em espaços de divertimento em vez de estarem estudando.

Entre os sintomas gerais, foram comuns cefaleia, dores nas costas, ansiedade, arritmia e insônia. Destacaram-se entre as queixas dos estudantes os sintomas e patologias digestivas: refluxo gastroesofágico, epigastralgia, pirose, náuseas, alteração de hábito intestinal e até mesmo o diagnóstico de gastrite. Os gatilhos mais frequentes foram estresse, privação de sono e eventos da vida diária ${ }^{24}$

O etilismo, referido por alguns estudantes, pode contribuir com os sintomas gastrointestinais. Já são bem conhecidas as ações tóxicas e deletérias do álcool sobre o fígado e o pâncreas, no entanto ele pode afetar todo o trato digestivo. No esôfago, o consumo de etanol pode contribuir para a exacerbação da doença do refluxo esofágico, uma vez que reduz o tono do esfíncter inferior do esôfago (EIE), aumenta a frequência de relaxamentos espontâneos do EIE e reduz a depuração esofágica. Além disso, o álcool por ser muito calórico e levar o indivíduo que dele faz uso a negligenciar o horário e a ingesta de qualidade de alimentos saudáveis ${ }^{25}$.

No que tange aos hábitos prejudicados de sono relatados pelos participantes deste estudo, sabe-se que o organismo, assim como as múltiplas funções fisiológicas, psicológicas e comportamentais, segue o ritmo circadiano, como temperatura corporal, liberação de corticosteroides e eletrólitos do soro e urinários, funções cardiovasculares, secreção de enzimas gástricas, número de leucócitos no sangue, força muscular, estado de alerta, humor e memória imediata e de longo prazo. Desse modo, os cursos de Medicina devem se preocupar com a qualidade do sono de seus estudantes, visto que, quando há prejuízo do sono, como se verificou nos resultados desta pesquisa, praticamente todo o metabolismo orgânico fica alterado, e esse fato pode prejudicar o desempenho dos alunos ${ }^{26}$. Em relação ao estresse, a literatura tem evidenciado plenamente a influência dele nos cursos de Medicina, confirmando os achados desta pesquisa.

Assim, dados parecem indicar que, antes da diplomação, o médico sofre prejuízos no domínio psicológico durante a graduação. Desse modo, esse assunto deve ser prioritário para os educadores da área de saúde e as autoridades sanitárias. Autores concluem que a qualidade de vida dos estudantes de Medicina, quando avaliada pelo instrumento World Health Organization Quality of Life (Whoqol-bref), sofre desgastes no domínio psicológico ao longo do curso médico ${ }^{27}$.

Em um estudo qualitativo, apontaram-se as seguintes estratégias para reduzir o estresse no decorrer do curso médico: valorização dos relacionamentos interpessoais e de fenômenos do cotidiano; equilíbrio entre estudo e lazer; organização do tempo; cuidados com a saúde, alimentação e sono; prática de atividade física; religiosidade; trabalhar a própria personalidade para lidar com situações adversas; procurar por assistência psicológica. Sugeriram-se ainda maior preocupação dos envolvidos na educação médica com a qualidade de vida do estudante de Medicina e o desenvolvimento de estratégias para promovê-la, ou para preparar o estudante para lidar com o estresse durante a formação médica ${ }^{28}$. Na unidade acadêmica na qual este trabalho foi realizado, houve preocupação em disponibilizar uma psicóloga para atender os discentes de Medicina, mas percebeu-se que apenas a contratação desse profissional não foi suficiente para aplacar o estresse desses estudantes.

Em consonância com as referências estudadas, a presente pesquisa evidenciou que o estudante de Medicina apresenta percepção de qualidade de vida diminuída relacionada à extensa grade curricular, o que dificulta o manejo do tempo. Ainda, há escassez na literatura científica nacional e internacional de estudos sobre as patologias digestivas que acometem o acadêmico de Medicina e que foram prevalentes entre o grupo pesquisado, além de outros sintomas gerais.

Quanto às limitações do artigo, a população definida para este estudo foi selecionada apenas do $1^{\circ}$ ao $8^{\circ}$ período. Com isso, as experiências vivenciadas pelos dois últimos anos, cruciais para a formação, foram deixadas para uma próxima 
abordagem. Além disso, cabe a ampliação do estudo com uma abordagem quantitativa, como a aplicação do questionário de qualidade de vida WHOQOL-100, da Organização Mundial de Saúde. Outra limitação é o fato de os pesquisadores e/ou pesquisados serem do mesmo ciclo social, o que por vezes inibiu algumas respostas mais completas e esclarecedoras por serem um tanto quanto constrangedoras.

\section{CONSIDERAÇÕES FINAIS}

Com base na pesquisa qualitativa realizada com estudantes de Medicina do $1^{\circ}$ ao $8^{\circ}$ período, por meio de grupos focais, inferiu-se que: se alimentam inadequadamente, não praticam atividades físicas regulares, se privam de sono e se queixam de sintomas gerais, como insônia, cefaleia, dores nas costas, ansiedade e arritmia, e sintomas gástricos, como diarreia, pirose, epigastralgia, refluxo. Os resultados coincidem com os trabalhos nacionais e internacionais sobre a qualidade de vida do discente de Medicina. Portanto, devem-se criar melhores infraestrutura e recursos educativos para aprimorar a qualidade de vida e fomentar hábitos e estilos de vida saudáveis, com especial atenção para a alimentação e a realização de adequada atividade física.

Os hábitos de vida interferem diretamente na qualidade de vida do indivíduo. Esse conceito faz referência ao bem-estar geral dos indivíduos e da sociedade, incluindo elementos de riqueza e emprego, de ambiente, de saúde física e mental, educação, diversão e sensação de pertencimento a um grupo social $^{29}$. Na população universitária, é de fundamental importância que os hábitos de vida sejam percebidos, já que os universitários se encontram em processo de formação para desenvolver projetos de vida profissional e pessoal ${ }^{30}$. Por isso, os resultados desta pesquisa podem despertar as instituições universitárias sobre a necessidade de intervir no curso de $\mathrm{Me}$ dicina, uma vez que neste trabalho se evidenciou considerável repercussão na qualidade de vida, sem exceção, entre estudantes dos sexos masculino e feminino em todos os períodos estudados, tanto por conta dos hábitos de vida - alimentação, lazer, etilismo, horas de sono - quanto da carga horária e da rotina da universidade.

O núcleo docente estruturante do curso de Medicina da UFAL está em processo de adequação às Diretrizes Curriculares Nacionais de 2014, sendo plausíveis futuras adaptações de acordo com as dificuldades mais encontradas nesta pesquisa. Seguindo a tendência mundial de currículo médico que aposta no autocuidado dos estudantes, espera-se formar profissionais mais capacitados a orientar seus pacientes, beneficiando também a população geral, afinal frequentemente os médicos são vistos pela população como modelos em uma comunidade e como cruciais agentes de mudança para promover a saúde e prevenir doenças ${ }^{31}$.

\section{REFERÊNCIAS}

1. Feodrippe A, Brandão MCF, Valente TCO. Qualidade de Vida de Estudantes de Medicina: uma Revisão. Rev bras educ med 2013;37(3)418-28. http://dx.doi.org/10.1590/ S0100-55022013000300014

2. Dyrbye L, Massie F, Eacker A, Harper W, Power D, Durning $S$, et al. Relationship Between Burnout and Professional Conduct and Attitudes Among US Medical Students. Jama 2010;304(11)1173-80. https://doi.org/10.1001/ jama.2010.1318

3. Dyrbye L, Thomas M, Massie F, Power D, Eacker A, Harper W, et al. Burnout and Suicidal Ideation among U.S. Medical Students. Ann intern med 2008;149(5)334-41. https:/ / doi.org/10.7326/0003-4819-149-5-200809020-00008

4. Backes D, Colomé JS, Erdmann RH, Lunardi VL. Grupo focal como técnica de coleta e análise de dados em pesquisas qualitativas. Mundo saúde 2011;35(4)438-42.

5. Fonseca R. Metodologia do Trabalho Científico. Curitiba: Iesde Brasil, 2012.

6. Henning A, Trevisan M. Análise de Conteúdo: Exemplo de Aplicação da Técnica para Análise de Dados Qualitativos. Brasília, 2013.

7. Silva A, Fossá MIT. Análise de conteúdo: exemplo de aplicação da técnica para análise de dados qualitativos. Qualitas 2015;16(1).

8. Peixoto A, Plácido A, Ribeiro C. Projeto Pedagógico do Curso de Medicina [on line]. Maceió UFAL, 2013 [capturado 20 dez 2018. Disponível em: http://www.ufal.edu. $\mathrm{br} /$ unidadeacademica / famed/graduacao/medicina / projeto-pedagogico/pcc-medicina-2013/view

9. Nisar N, Qadri MH, Fatima K, Perveen S. Dietary habits and life style among the students of a private Medical University Karachi. J pak med assoc 2009;59(2)98-101.

10. Hage C, Sayegh J, Rizk GA. Health habits and vaccination status of Lebanese residents: are future doctors applying the rules of prevention? J med liban 2010;58(2)91-6.

11. Becerra-Bulla F, Vargas-Zarate M. Estado nutricional y consumo de alimentos de estudiantes universitarios admitidos a nutrición y dietética en la Universidad Nacional de Colombia. Rev salud pública 2015;17(5)762-75. http:/ / dx.doi.org/10.15446/rsap.v17n5.4357

12. Bulla FB, Villate GP, Zárate MV, Barrios SV, Cetina MR. Estilos de vida de estudiantes universitarios admitidos al pregrado de la carrera de medicina, Bogotá 2010-2011. Rev facultad de medicina 2014;62(1Sup.)S51-6. 
13. Oliveiras-López M, Nieto Guindo P, Agudo Aponte E, Martínez Martínez F, López García de la Serrana H, López Martínez MC. Evaluación nutricional de una población universitaria. Nutr hosp 2006;21(2)179-83.

14. Kresic G, Kendel Jovanović G, Pavicić Zezel S, Cvijanović $\mathrm{O}$, Ivezić G. The effect of nutrition knowledge on dietary intake among Croatian university students. Coll antropol 2009;33(4)1047-56.

15. Jovanović GK, Kresić G, Zezelj SP, Mićović V, Nadarević VS. Cancer and Cardiovascular Diseases Nutrition Knowledge and Dietary Intake of Medical Students. Coll antropol 2011;35(3)765-74.

16. Silva LVR, Malbergier A, Stempliuk VA, Andrade AG. Fatores associados ao consumo de álcool e drogas entre estudantes universitários. Rev saúde pública 2006;40(2)280-8. http:/ /dx.doi.org/10.1590/S0034-89102006000200014

17. Barbosa FL, Barbosa RL, Barbosa MCL, Aguiar DL, Figueiredo IA, Ribeiro AC, et al. Uso de Álcool entre Estudantes de Medicina da Universidade Federal do Maranhão. Rev bras educ med 2013;37(1)89-95. http:/ /dx.doi. org/10.1590/S0100-55022013000100013

18. Borini P, Oliveira CM, Martins MG, Guimarães RC. Padrão de uso de bebidas alcoólicas de estudantes de medicina (Marília, São Paulo). J bras psiquiatr 1994;2(43)93-103.

19. Rodrigues AP, Oliveira AS, Zaleski EGF, Arantes SL. Avaliação do nível de propensão para o desenvolvimento do alcoolismo entre estudantes do curso de graduação em enfermagem da Universidade Católica Dom Bosco. SMAD 2007;3(1)110. https:/ /doi.org/10.11606/issn.1806-6976.v3i1p01-10

20. Araújo M, Lima A, Alencar A, Araújo T, Fragoaso L, Damasceno M. Avaliação da qualidade do sono de estudantes universitários de Fortaleza-CE. Texto Contexto Enferm 2013;22(2)352-60. http:/ /dx.doi.org/10.1590/S010407072013000200011

21. Cardoso H, Bueno F, Mata J, Alves A, Jochims I, Vaz Filho I, et al. Avaliação da qualidade do sono em estudantes de Medicina. Rev bras educ med 2009;33(3):349-55. http:// dx.doi.org/10.1590/S0100-55022009000300005

22. Purim KSM, Guimarães ATB, Titski ACK, Leite N. Privação do sono e sonolência excessiva em médicos residentes e estudantes de medicina. Rev col bras cir 2016;43(6)43844. http:/ / dx.doi.org/10.1590/0100-69912016006005

23. Frank E, Carrera J, Elon L, Hertzberg V. Basic Demographics, Health Practices, and Health Status of U.S. Medical Students. Am j prev med 2006;31(6)499-505. https://doi. org/10.1016/j.amepre.2006.08.009
24. Perveen I, Parvin R, Saha M, Bari S, Huda MN, Ghosh MK. Prevalence of Irritable Bowel Syndrome (IBS), Migraine and Co-Existing IBS- Migraine in Medical Students. J clin diagn res 2016;10(11)OC09-OC13. https: / / doi. org/10.7860/JCDR/2016/20900.8832

25. Figuinha FCR, Fonseca FL, Moraes-Filho JPP. Ações do álcool sobre o esôfago, estômago e intestinos. Rev bras med 2005;62(1/2)10-6.

26. Vieira ACB. Agravos à saúde do trabalhador da área da saúde, com ênfase nas alterações do ciclo sono-vigília, ligados ao trabalho noturno. Conselheiro Lafaiete; 2009. Doutorado [Tese] -Universidade Federal de Minas Gerais.

27. Alves JGB, Tenório M, Anjos AGD, Figueroa JN. Qualidade de vida em estudantes de Medicina no início e final do curso: avaliação pelo Whoqol-bref. Rev bras educ med 2010;34(1)91-6. http://dx.doi.org/10.1590/S010055022010000100011

28. Zonta R, Robles ACC, Grosseman S. Estratégias de enfrentamento do estresse desenvolvidas por estudantes de medicina. Rev bras educ med 2006;30(3)147-53. http:// dx.doi.org/10.1590/S0100-55022006000300005

29. Gregory D. The Quality of Life. The Dictionary of Human Geography. Oxford: Wiley, 2009.

30. Wanden-Berghe C, Martín-Rodero H, Rodríguez-Martín A, Novalbos-Ruiz JP, Victoria EM, Sanz-Valero J, et al. Calidad de vida y sus factores determinantes en universitarios españoles de Ciencias de la Salud. Nutr hosp 2015;31(2)95258. http:/ / dx.doi.org/10.3305/nh.2015.31.2.8509

31. Talwar K, Grover A, Thakur J. Role of Medical Education in Preventing and Control of Noncommunicable Diseases in India? Indian j community med 2011;36(Supl.1):S63-S66. https:/ /dx.doi.org/10.4103\%2F0970-0218.94711

\section{CONTRIBUIÇÃO DOS AUTORES}

Os autores participaram igualmente de todas as etapas de elaboração deste artigo.

\section{CONFLITO DE INTERESSES}

Não há conflito de interesses.

O financiamento foi próprio.

\section{ENDEREÇO PARA CORRESPONDÊNCIA}

Angela Maria Moreira Canuto Mendonça - Campus A.C. Simões, Faculdade de Medicina, Av. Lourival Melo Mota, s/n - Tabuleiro do Martins, AL, 57072-900, Maceió-AL, Brasil. angela_canuto@uol.com.br 